\title{
Physical and Functional Properties of Antibacterial Nylon/Bamboo Polyester/Stainless Steel Wrapped Yarns and Knitted Fabrics
}

\author{
Zhi-cai Yu ${ }^{1,2, a}$, Jian-Fei Zhang ${ }^{1, b}$, Ching-Wen Lou ${ }^{3, c}$, Jia-Horng Lin ${ }^{4,5,6, d_{*}}$ \\ ${ }^{1}$ School of Textiles, Tianjin Polytechnic University, Tianjin 300387, China \\ ${ }^{2}$ Functional Textile Materials Laboratory of Eastern Liaoning University, School of Chemical \\ Engineering and Material Science, Eastern Liaoning University, Dandong 118003, China
}

${ }^{3}$ Institute of Biomedical Engineering and Material Science, Central Taiwan University of Science and Technology, Taichung 40601, Taiwan

${ }^{*}$ Laboratory of Fiber Application and Manufacturing, Department of Fiber and Composite Materials, Feng Chia University, Taichung 40724, Taiwan

${ }^{* 5}$ School of Chinese Medicine, China Medical University, Taichung 40402, Taiwan

*6 Department of Fashion Design, Asia University, Taichung 41354, Taiwan

aEmail:yuzhicaicai@163.com, ${ }^{\mathrm{b} E m a i l}$ :zhangjianfei1960@126.com, ${ }^{\mathrm{C} E m a i l}$ :Ifam3405@gmail.com, d*Email: jhlin@fcu.edu.tw

Keywords: Stainless Steel Wire, Bamboo Charcoal Polyester, Metal Composite Yarns, Electromagnetic Shielding Effectiveness, Water Evaporation Rate

\begin{abstract}
There are derivative many problems from electromagnetic (EM) wave radiation with the development of communication technique. To protect the human body from the EM radiation, in this study, the antibacterial nylon (AN)/ bamboo charcoal polyester (BC-PET)/ stainless steel wire (SSW) metal composite yarns was produced on a hollow spindling spindle machine. In this study, we used the SSW as the core yarn, the AN and BC-PET as the outer and inner wrapped material, respectively. The wrapped amounts were varied from 6.5-11 turns/cm. Then, the AN/BC-PET/SSW metal composite yarns were woven into knitted fabrics on a circular knitting machine. Moreover, we also investigated the electromagnetic shielding effectiveness (EMSE) and water evaporation rate of the fabricated metal composite knitted fabrics. Optimum EMSE -30dB was obtained at the incident frequency of $0.54 \mathrm{GHz}$.
\end{abstract}

\section{Introduction}

In recent years, the usage of electrical and electronic devices has grown rapidly. These electrical equipments will emit electromagnetic energy in their normal operation [1]. Hence, the human body will exposes to electromagnetic field at everywhere, especial close to communication apparatus. If the EM waves could not be shielded effectively, they not only cause interference with other electrical devices but may also have great physical harm to the human tissue [2-3]. At present, there is a growing body of scientific evidence that long-term exposure to EM wave will have a potential health hazards such as brain tumours and sleep problem etc [4].Thus, there is a growing need for suitable materials to protect the human body from EM radiation.

Traditionally, metal is used to as EM shielding materials because of its conductivity and permeability. However, the shortcoming in terms of heavy and corrosion problems limit its wide application. In recently years, conductive metal composite fabrics have started to use as EM shielding materials to produce EM shielding personal protective clothing due to their desirable flexibility and lightweight properties.

In this study, we aimed to design a type of antibacterial nylon (AN)/bamboo charcoal polyester (BC-PET)/stainless steel wire (SSW) wrapped yarns and theirs knitted fabrics. The effect of wrapped amount on the breaking strength, elongation of the produced wrapped yarns was investigated. In addition, we also assess the electromagnetic shielding and drying properties. 


\section{Experimental}

\section{Wrapped yarns production by hollow spindle spinning}

These AN/BC-PET/SSW wrapped yarns were produced on a hollow spindle spinning machine with SSW as core yarn, the AN (S-direction) and BC-PET (Z-direction) as the inner and outer wrapped yarns, respectively. The wrapped amounts of these produced wrapped yarns were 6.5, 8.0, 9.5, and 11.0 turns/cm. Hence, four types of wrapped yarns W-6.5, W-8, W-9.5 and W-11 were produced, respectively. 150d/144f BC-PET was obtained from Hua Mao Co. Ltd. The $40 \mu \mathrm{m}$ stainless steel wires were purchased from King's Metal Fiber Technology Co., Ltd. 150d/144f AN filaments were provided by Industrial technology research institute, Taiwan.

\section{Production of metal composite knitted fabric samples}

The knitted fabrics used in this study were successfully produced using a 20-gauge circular knitting machine. Metal composite knitted fabrics with a single jersey construction were produced using these wrapped yarns with different wrapping numbers, respectively. As a result, four kind of knitted fabrics K-6.5, K-8.0, K-9.5 and K-11.0 were produced in this study. The produced metal composite knitted fabric was shown in Figure 1.From the image of the W-8

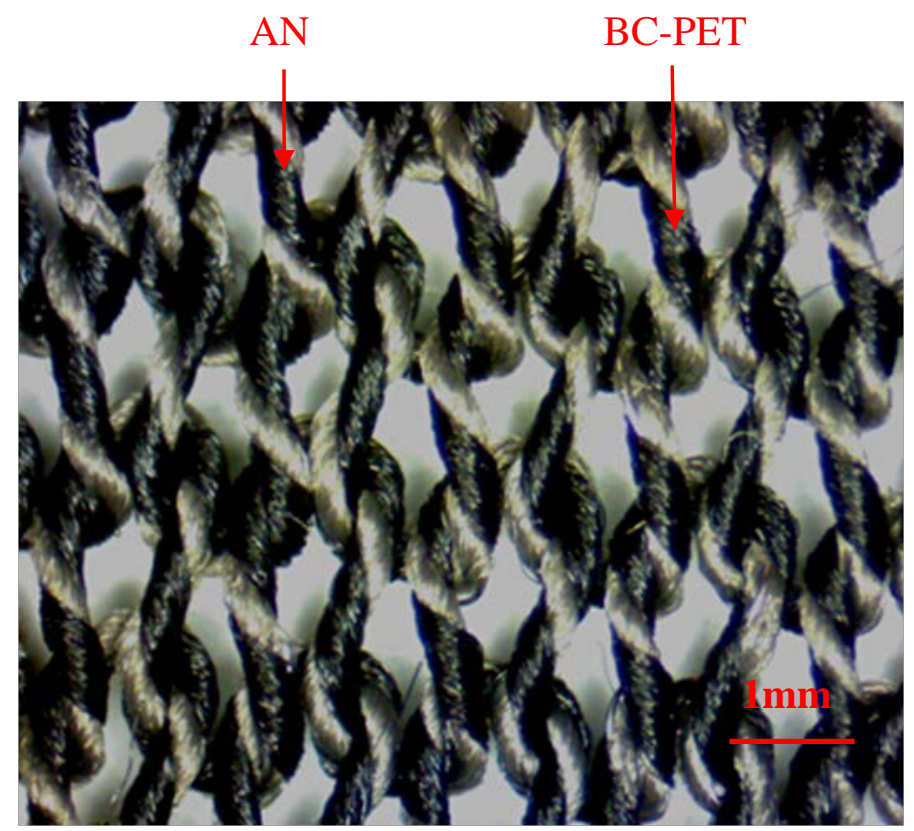

Fig.1. Illustration of AN/BC-PET/SSW metal composite knitted fabric W-8.0.

\section{Testing EMSE of metal composite knitted fabric}

A coaxial transmission line method as per ASTM D4935 was used to test the metal composite knitted fabric using a network analyser (HP Agilent Co., Ltd., 8753B) and a test fixture (Electro-Metrics, Inc., EM-2107A) as shown in Figure 2.The range for frequency sweep was varied from 30 to $3000 \mathrm{MHz}$.

\section{Testing drying ability of the metal composite knitted fabrics}

The dry ability was assessed by using the calculating the water evaporation rate (WER). A drop of water about $0.05 \mathrm{~g}$ was dropped on the surface of the fabric using a pipette. Then we weight the mass change $\Delta \mathrm{m}$ of the fabric after $12 \mathrm{~min}$. Finally, WER was calculated using the following equation:

$$
\text { WER }(\%)=\frac{\Delta \mathrm{m}}{0.05} \times 100 \%
$$




\section{Results and discussion}

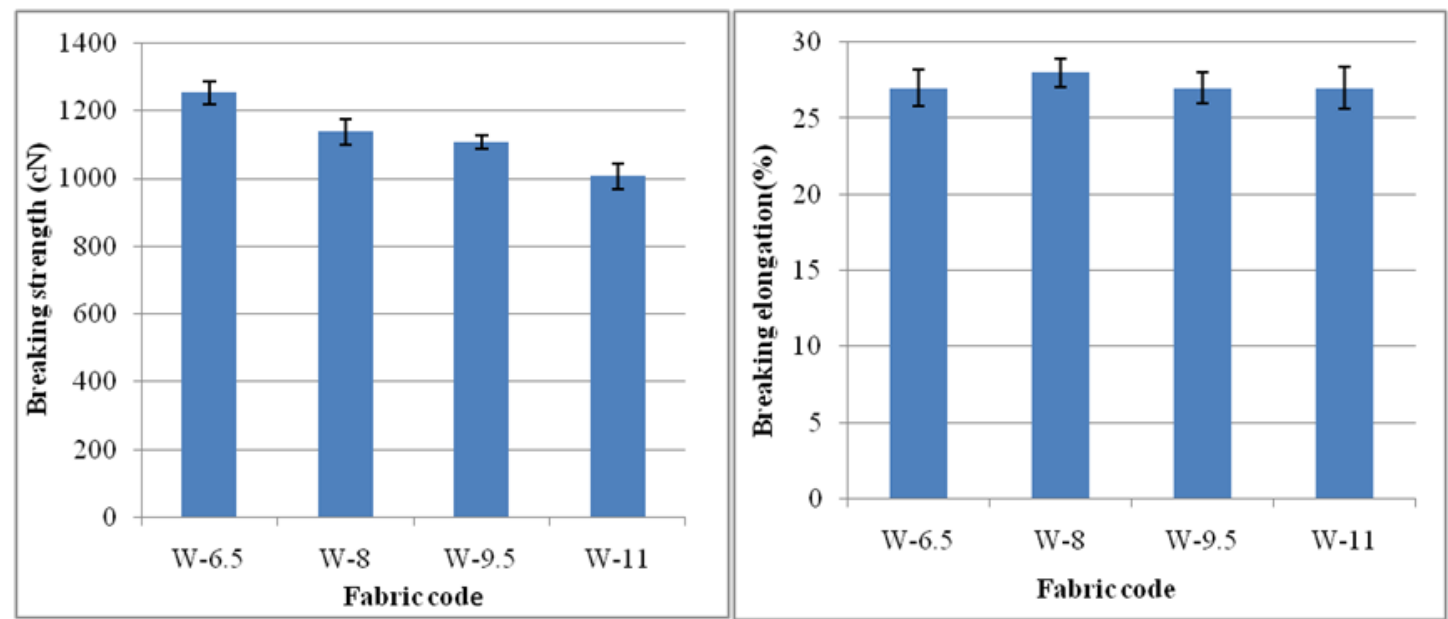

Fig.2. Effect of wrapped amounts on breaking strength of the wrapped yarns

Fig.3. Effect of wrapped amounts on breaking elongation of the wrapped yarns
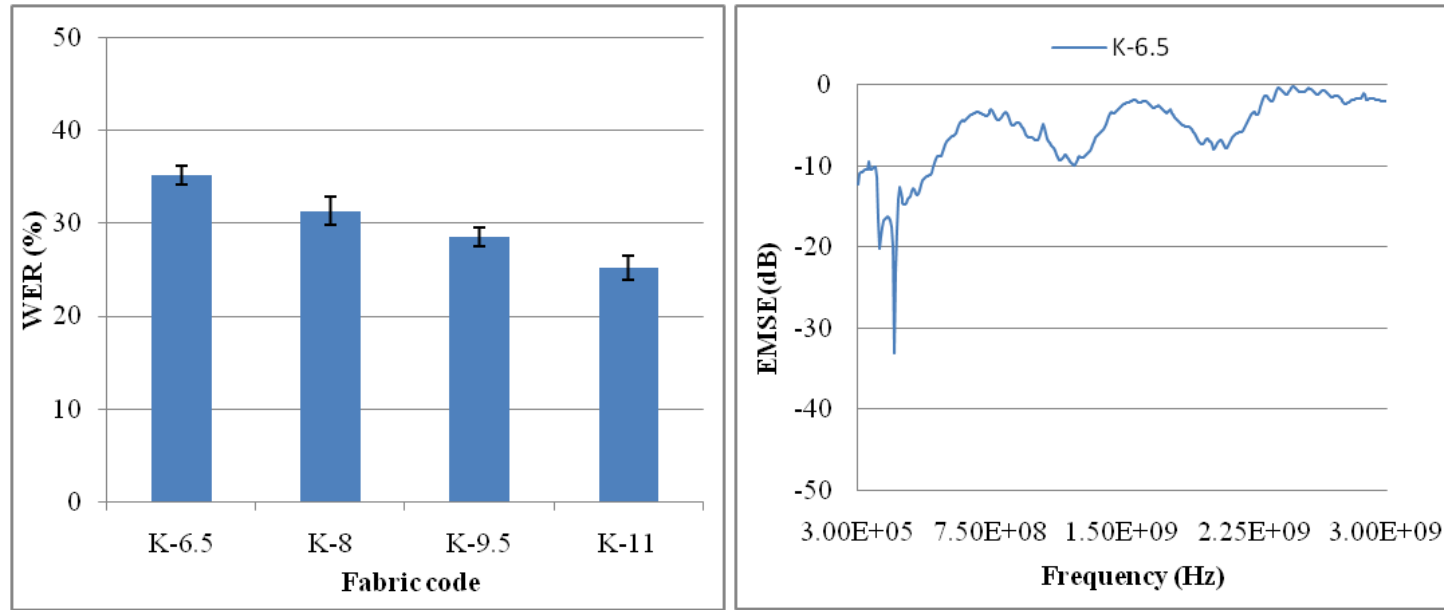

Fig.4. Effect of wrapped amounts on

Fig. 5. EMSE of the knitted fabric K-6.5 WER of the metal composite knitted fabrics.

Fig.2 indicates that with the increase of wrapped amount, the breaking strength of AN/BC-PET/SSW metal composite yarns would decrease. This was due to the wrapped angles would increase which resulting the distraction of axial strength decrease. Hence, the breaking strength decreased with increasing wrapped amounts.

Fig.3 shows the breaking elongation of these produced wrapped yarns. From Fig.4, it was found that the variation of the elongation was not significant. This was because the AN and BC-PET yarns had similar breaking elongation. However, it should be noted that the wrapped yarn W-8 displayer the highest breaking strength among these produced metal composite yarns. This was due to the wrapped materials displayed the highest slippage property when the wrapped amount was 8 turns/cm.

WER property of the metal composite knitted fabrics should also be assed when they are wore as EM shielding protective clothing. High WER of the fabric could effectively evaporate the absorbed sweat from the skin. Therefore, higher WER will give a better comfort for the wearers. From the Figu.4, it was found that the metal composite knitted fabric K-6.5 displayed the highest WER, whereas the fabric K-11 showed the lowest WER. This was due to with the increase of the wrapped amounts, the outer wrapped materials would increase, thus the BC-PET would be more covered by the AN yarns. Therefore, the absorbed water in the produce knitted fabric would evaporate difficulty with the increase of wrapped amounts of the metal composite yarns.

In this research, the scan frequency varied from $300 \mathrm{~K}$ to $3 \mathrm{GHz}$ was used to assess the EMSE of 
the produced metal composite knitted fabrics. Considering the breaking strength of the metal composite yarns and the WER of the metal composite knitted fabric, we chose fabric K-6.5 as a representative to assess the EMSE of fabric. From Fig. 5, it was found that fabric K-6.5 showed obviously EM shielding ability. This finding was due to the presence of SSW in the woven fabric that caused the effective absorption and reflection of the EM wave. In this study, the highest EMSE would reach up to $-30 \mathrm{~dB}$ when the incident frequency was $0.54 \mathrm{GHz}$.

\section{Conclusions}

In this research, we successfully fabricated the AN/BC-PET/SSW metal composite yarns on a hollow spindle spinning machine. These fabricated metal composite yarns were then woven into knitted fabric by using a 20 gauge circular knitting machine.

Breaking strength of these fabricated metal composite yarns would decrease with the increase of the wrapped amount. However, it should be note that all the produced AN/BC-PET metal composite yarns displayed greatly breaking strength and higher than $1000 \mathrm{cN}$.

In additional to the breaking, we also found that the WER of the produced knitted fabric would also decrease with the increase of the wrapped amount of the metal composite yarns. The presence of SSW obviously improved the EMSE. In this study, the EMSE was reach up to 0--10dB in most frequency range.

\section{Acknowledgements}

The authors are grateful to the Laboratory of Fiber Application and Manufacturing, Feng Chia University, for providing research materials, laboratory equipment and financial support (NSC-103-2221-E-035-028).

\section{References}

1. Rajendrakumar and G Thilagavathi. Journal of Industrial Textiles.42 (2013), p400

2. V. Safarova and J. Militky. Textile Research Journal,0 (2014), p400.

3.Williams T. EMC for product designer, 3 rd edn.Boston, MA: Newnes, 2001

4. M. A. Stuchly. Electromagnetic fields and health potentials 41993, p.34. 